\title{
Research on Automotive Engine Common Fault Diagnosis and Maintenance Measures Analysis
}

\author{
Bin Wang \\ Wuxi Vocational Institute of Commerce, Wuxi, China, 214153 \\ 45078604@qq.com
}

Keywords: Automotive Engine; Common Fault; Diagnostic Maintenance

\begin{abstract}
With the development of science and technology and economic progress, the car as people's conventional means of transport, a lot of people into the daily life of people. In the overall structure of the car, the engine is the most burden of the region, is the most common failure of the components. The normal operation of the car engine is an important condition for ensuring that the vehicle is running normally. When the car engine failure, we should adopt a more reasonable inspection measures. Only to determine the cause of the car engine failure, and thus according to the reasons for the corresponding repair, and ultimately ensure the good operation of the car engine to ensure the safety of road driving. In the overall structure of the car engine is a key component, shorten the maintenance time, for improving the engine economy, power and environmental protection is of great significance.
\end{abstract}

\section{Introduction}

The quality of the car engine itself is not only related to the life of the car, but also has an important impact on the environment. Therefore, to increase the vehicle engine troubleshooting and maintenance research efforts, not only can effectively improve the quality of the work of the car engine, but also improve its efficiency. As a result of different reasons, the car engine in the type of failure also showed a variety of characteristics. The engine can't start, the normal operation of the engine not only need to meet enough ignition voltage and energy, but also need to ensure that the ignition time is appropriate, the air-fuel ratio of precision and cylinder pressure normal. The cause of the failure of the engine can't be initiated from the ignition system, fuel systems and mechanical systems to be excluded.

The sound emitted by the engine can be divided into three categories, namely, normal, benign and malignant. Lack of oil, the engine temperature is too high, the cylinder pad burned, improper ignition timing and crankshaft connecting rod bearings and other reasons are likely to cause the engine abnormal sound. Engine abnormal sound can increase the mechanical loss to a certain extent, making the launch life reduced. Engine lack of cylinder problems in the actual maintenance work is more common to ensure that the engine will not appear missing cylinder factors are three parties, respectively, for the ignition energy is sufficient, the air-fuel ratio is appropriate, mechanical condition is good, as long as one of the problems, may cause The engine appears missing cylinder problem, resulting in engine power reduction, emissions exceeded the problem ${ }^{[1]}$.

Diagnosis can monitor the fault parameters compared with the normal parameters, in the choice of contrast parameters, combine the actual situation of the car to avoid errors, is not conducive to maintenance tasks. Parameters should also maintain a comprehensive, such as the car running every kilometer fuel consumption, vibration, the noise generated, etc., are within the choice of parameters within the judge. These parameters are also required for the maintenance phase, the parameters are adjusted properly, and car failure was completely excluded. With the information technology, the maintenance of faulty vehicles, to achieve real-time data transmission, equipment detection data will be transmitted directly to the computer, and in the system to complete the task of analysis, while saving time at the same time the accuracy of the results significant ascension. 


\section{Basic Steps of Engine Fault Diagnosis}

At present, with the continuous improvement of science and technology, modern car performance has been great improved, and the car's braking system has become more scientific and advanced. In this case, the motor vehicle engine fault diagnosis and maintenance has become more difficult, which requires vehicle maintenance personnel to continuously improve their business level. When the engine fails, the maintenance staff can't rush to handle, but should do more investigation, observation and testing, only to be aware of it, can calmly deal with the failure. When the engine fails, the driver should take emergency measures to stop the system to run, protect the scene, and record the status of failure to facilitate the exchange with the maintenance staff ${ }^{[2]}$. Fig. 1 shows basic steps of engine fault diagnosis.

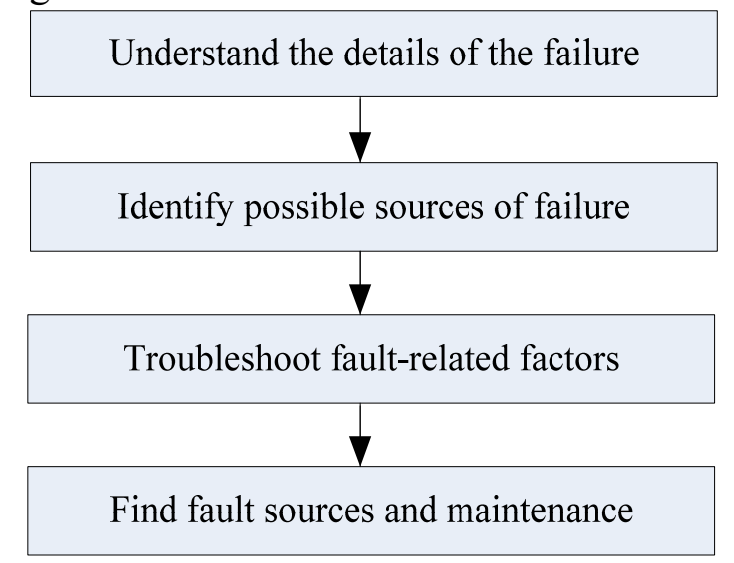

Fig. 1.The basic steps of engine fault diagnosis

Fault diagnosis steps generally include the following steps: First, the driver to understand the details of the failure, which is an important basis for maintenance personnel maintenance. Car maintenance personnel must pay attention to this point, and to analyze the possible causes of failure to reduce the scope of the fault in order to quickly and accurately determine the source of the fault. Secondly, after thorough analysis of the fault, the fault phenomenon and the launch of the system components to determine the possible fault source. Some failures arise only a small part of the problem, easy to determine the direction of the fault to find the direction.

Some causes of failure involve multiple mechanical and electronic components. It is difficult to determine the direction of the fault source by a simple method. Check the relevant information on engine maintenance to determine the various factors related to the failure, list a number of search directions and one by one to conduct investigation. According to the principle of fault finding to find the source of the fault, fault search generally follow the "first want to do, after the first electrical appliances, the first static after the move, the first external after the internal, first simple and complex, the first general after the special" principle. In the fault diagnosis process, you can first understand the engine failure phenomenon, and then analysis, and finally based on the results of the analysis and fault search for fault repair ${ }^{[3]}$.

\section{Analysis of Automobile Engine Common Faults}

The engine is a component that provides power for the car. It is the machine that converts the chemical energy of the fuel into the mechanical energy of the piston movement and outputs it to the outside. It is the heart of the car and provides power for the car. Its working principle is to liquid, gas energy or new energy into heat, and then heat into mechanical energy and external output power.

The engine start problem is one of the more common faults of the engine. There are many reasons for the engine startup problem, such as car spark plug problems, or car engine current line fault problems, which will cause the engine to start the fault and other failures lead to the car can' $t$ stop running. Engine noise problem, refers to the car in the usual driving process, due to some reasons leading to the engine internal bad sound. There are many causes of noise problems in the 
car engine, such as loosening or damage to the relevant parts inside the engine, the leakage of the relevant circuits in the engine and the valve failure, which can cause varying degrees of noise problems and affect the use of the vehicle ${ }^{[4]}$.

Cylinder is an important structure of engine, so the piston is the main work unit for rapid operation. If the cylinder problems, it will cause the car fuel consumption increases, lack of car driving power, and even car engine burns and other accidents. Therefore, the cylinder problem is the main problem of the engine. The cause of the cylinder failure is mainly caused by the cylinder airtight damage, resulting in leakage, leakage, or cylinder piston shaft appeared bad movement wish. Engine blasting and knocking phenomenon is more common, mainly refers to the exhaust of the exhaust pipe has a violent phenomenon, mainly because the car engine gasoline combustion is not sufficient or the car engine carburetor failure caused. In general, the engine foam and knocking phenomenon indicates that the engine have a serious internal operation problems, and if the gasoline combustion is not sufficient to bring more serious gasoline consumption, and even affect the life of the car.

\section{Engine Fault Maintenance Measures}

The automobile is indispensable transportation equipment in people's daily life. At present, there are obvious progress in the automobile production and processing technology. The automobile product is also advanced, and the problem of vehicle fault maintenance is gradually highlighted. Maintenance technology should be combined with product performance to make progress, so as to ensure the effectiveness of maintenance links, maintenance technicians during the need to judge the cause of the failure, common failures due to maintenance experience, able to complete the task in a short time ${ }^{[5]}$. Fig.2 shows common engine trouble shooting measures.

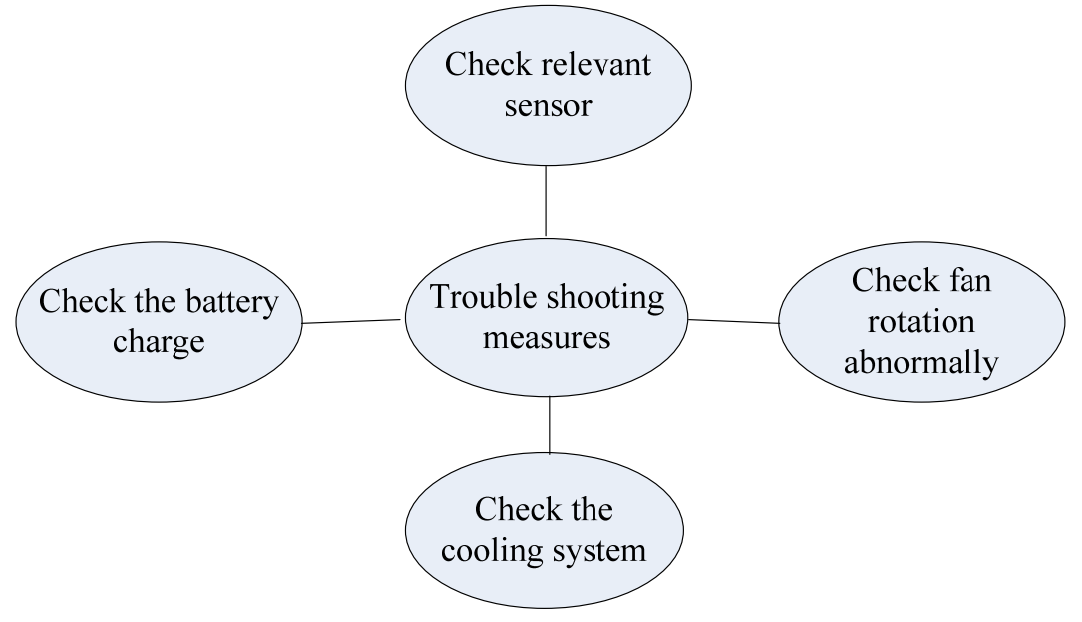

Fig. 2. The common engine trouble shooting measures

The main character of this failure is the car in the normal driving process suddenly turn off, in this case, wait for the engine cooling after the start again, if it is not normal operation, and the car driving panel fault warning light, you can determine Failure of the cooling system. The treatment is: first check the coolant is sufficient, and then check the ignition controller and voltage is normal. It is noteworthy that the long-term operation of the car engine in the high temperature state, it is very easy to lead to other aspects of the car also appears to be damaged, so maintenance personnel must be careful and meticulous work, the only way to identify the reasons for the right medicine. Therefore, you should first check the battery power is sufficient, if not enough to replace the battery in time. If the battery check is correct and the fault is still there, we need to check the engine oil system is the case of insufficient oil supply, as appropriate to be maintained.

Under normal circumstances, the car in the process of starting or driving noise, first of all according to the noise of the outgoing location and different degrees to determine which part of the parts there is a problem. Such as the inspection found that the ignition system is faulty, first check the engine fan blade rotation is abnormal, and take appropriate measures to repair; if found for some 
reason the engine cylinder internal abnormal sound, you need to wait for the engine after cooling. In accordance with a certain order to open the engine, find the point of failure, and then take the appropriate approach. As the cylinder to stop working or the use of fuel failure, easy to lead to the car exhaust pipe blasting phenomenon, first check the car engine circuit is intact, the relevant sensors and other equipment is running normally, in ensuring the normal working conditions of the spark plug, observe the exhaust pipe If the combustion is not sufficient, the concentration of the mixed gas in the cylinder is too high, and the engine is in the overload operation. In this case, if the fuel is not in the air, Replace the fuel and check it again.

\section{Conclusions}

Automobile engine is an important power unit of the car, due to the lack of daily maintenance of the car and the use of methods and improper maintenance and other issues, resulting in the car engine is prone to the corresponding failure, thereby reducing the car's life, affecting the car's regular use The Maintain the normal work of the engine is the safety of motor vehicles can be stable and stable. With the rapid development of science and technology, modern automobile technology has also made rapid progress, and the engine failure has become a variety of forms of failure. Therefore, the maintenance of the car engine will adhere to the "specific problem specific analysis" principle, so as to effectively find the cause of the failure, targeted to do the maintenance work. The scientific and reasonable maintenance and maintenance of the automobile engine is of great significance to improve the driving performance of the vehicle and the service life of the engine.

\section{References}

[1] WANG Shu-zhen. Study on related technology of vehicle engine fault repair [J]. China Machinery, 2015 (8): 27-28.

[2] XU Yong. Application Analysis of Vehicle Engine Failure Maintenance Technology [J]. Technology Economy Market, 2014: (4): 15-16.

[3] ZHAO Xian hua. Study on common problems and maintenance methods of automobile engine [J]. Military and civilian technology and products, 2015 (2): 33.

[4] Luo Yi-jun. Investigate automobile engine fault repair techniques [J]. Shandong Industrial Technology, 2016 (3): 14-15.

[5] Chen Xiu-yu. Theory and methods of automobile engine fault diagnosis [J]. Science and Technology Information, 2016 (4): 54-55. 